\title{
Projected health impact of post-discharge malaria chemoprevention among children under the age of five years with severe malarial anaemia in Africa: a modelling analysis
}

Lucy C Okell, ${ }^{1 *}$ Titus K. Kwambai, ${ }^{2,3}$ Aggrey Dhabangi ${ }^{4}$, Carole Khairallah ${ }^{3}$, Thandile NkosiGondwe $^{5,6}$, Robert Opoka ${ }^{4}$, Andria Mousa, ${ }^{1}$ Melf-Jakob Kühl ${ }^{7}$, Tim C. D. Lucas ${ }^{8}$, Richard Idro ${ }^{4}$, Daniel J. Weiss ${ }^{9,10}$, Matthew Cairns ${ }^{11}$, Feiko O ter Kuile ${ }^{3}$, Kamija Phiri ${ }^{5,6}$, Bjarne Robberstad ${ }^{7}$, Amani Thomas Mori*7,12

1. MRC Centre for Global Infectious Disease Analysis, Department of Infectious Disease Epidemiology, Imperial College, London W2 1PG, UK.

2. Centre for Global Health Research (CGHR), Kenya Medical Research Institute (KEMRI), Kisumu, Kenya

3. Department of Clinical Sciences, Liverpool School of Tropical Medicine (LSTM), Liverpool, United Kingdom

4. College of Health Sciences, Makerere University, Kampala, Uganda.

5. Kamuzu University of Health Sciences, Blantyre, Malawi

6. Training and Research Unit of Excellence, Blantyre, Malawi

7. Section for Ethics and Health Economics, Department of Global Public Health and Primary Care, University of Bergen, P.O. Box 7804, 5020 Bergen, Norway

8. Big Data Institute, University of Oxford, Oxford, UK

9. Malaria Atlas Project, Telethon Kids Institute, Perth Children's Hospital, 15 Hospital Avenue, Nedlands, Australia.

10. Curtin University, Bentley, Australia

11. International Statistics and Epidemiology Group, London School of Hygiene and Tropical Medicine, London, UK.

12. Chr. Michelsen Institute P.O. Box 6033, N-5892 Bergen, Norway.

*Contributed equally, corresponding authors

Lucy Okell, MRC Centre for Global Infectious Disease Analysis, Department of Infectious Disease Epidemiology, Imperial College London, UK. Email: 1.okell@imperial.ac.uk 
Amani Thomas Mori, Section for Ethics and Health Economics, Department of Global Public Health and Primary Care, University of Bergen, P.O. Box 7804, 5020 Bergen, Norway. E-mail: pax_amani@yahoo.com

\begin{abstract}
Background

Children discharged from hospital after recovery from severe malarial anaemia (SMA) are at high risk of readmission and death in subsequent months. Clinical trial results show that three months of post-discharge malaria chemoprevention (PMC) with dihydroartemisinin-piperaquine reduces this risk.
\end{abstract}

\title{
Methods
}

We developed a compartmental mathematical model describing the daily post-discharge incidence of uncomplicated and severe malaria requiring readmission among a cohort of 0-5 year-old children. We fitted the model to PMC and placebo groups from nine trial hospitals in areas of moderate-to-intense malaria transmission in Uganda and Kenya using Bayesian methods. The cohort model was then embedded within a full population model of SMA to predict impact of PMC across malaria-endemic African countries.

\section{Findings}

The incidence of hospitalised malaria episodes during the first 6 months post-discharge is estimated to be $\sim 23-60$ times higher than the average for children of this age, depending on transmission intensity. We estimate that repeat SMA episodes within 6 months of the original episode constitute $18-27 \%$ of all SMA episodes in high transmission settings. In the 20 highestburden countries in Africa, only 2-5 children need to be given PMC to prevent one hospitalised malaria episode, and less than 100 to prevent one death. If all hospitalised cases access PMC, we estimate that 36,000 (range 16,000-82,000) malaria-associated readmissions could be prevented annually, depending on the proportion accessing hospital care.

\section{Interpretation}


PMC has high potential impact per child treated across a range of epidemiological settings in Africa.

\section{Funding}

Research Council of Norway

\section{Introduction}

Severe malarial anaemia (SMA) still contributes substantially to childhood mortality and morbidity in malaria-endemic countries in Africa. P. falciparum malaria causes anaemia by triggering severe haemolysis of erythrocytes and suppression of erythropoiesis. ${ }^{1}$ In highly endemic areas, around one-third of all hospitalised children may be severely anaemic $(\mathrm{Hb}<5 \mathrm{~g} / \mathrm{dL})^{2,3}$ and severe anaemia may contribute to around half of all malaria-attributed deaths. ${ }^{4}$ While malaria transmission has declined in many countries in the past two decades, highly endemic conditions persist in parts of sub-Saharan Africa. 70\% of global malaria deaths occurred in just ten countries in Africa in 2020. ${ }^{5}$ These countries are now the focus of the WHO High Burden to High Impact (HBHI) initiative, aimed at accelerating progress in malaria control in the hardest-hit areas. ${ }^{5}$

Between 0.4 and $13 \%$ of children with severe anaemia in low and lower-middle-income countries die during the acute hospitalisation phase, but those who survive also remain at high risk of readmission and death following hospital discharge despite having received appropriate care. ${ }^{6} \mathrm{~A}$ recent meta-analysis found that the odds of death during the first six months after discharge is $72 \%$ higher than during hospitalisation and over two times higher than those admitted with other conditions without severe anaemia. ${ }^{7}$ Full haematological recovery from malaria may take around six weeks following treatment, and further malaria re-infections during this period substantially increase the risk of recurrent severe anaemia or death. ${ }^{8,9}$ However, no specific interventions have been widely implemented to tackle this large post-discharge burden of morbidity and mortality among SMA patients. 
Post-discharge malaria chemoprevention (PMC), consisting of full treatment courses of longacting antimalarials administered at pre-determined time intervals after discharge from hospital among children recently admitted with severe anaemia, can reduce the high post-discharge malaria morbidity and mortality. In Malawi, three months of PMC with monthly artemether-lumefantrine achieved a protective efficacy of $41 \%$ against the risk of death or all-cause readmission and $49 \%$ against clinical malaria in the first 3 months post discharge. ${ }^{10}$ More recently, a multicentre trial in Uganda and Kenya showed that three months of PMC with monthly dihydroartemisininpiperaquine (DP) reduces the risk of deaths or all-cause readmissions by $70 \%$ and hospitalised malaria episodes by $87 \%$ during the same period. ${ }^{11}$

As malaria-endemic countries decide whether to implement PMC, there is a need to understand the potential epidemiological impact in settings with varying transmission intensity to support economic evaluation analysis, and planning how PMC could contribute to initiatives such as the HBHI programme. This study characterises the burden of post-discharge malarial disease and estimates the demand for PMC and its potential public health impact in Africa.

\section{Methods}

\section{Post-discharge data}

Data were from a PMC trial conducted in 2016-2018. ${ }^{11}$ This randomised, placebo-controlled trial was conducted in nine hospitals in areas with moderate-to-intense perennial malaria transmission in Kenya and Uganda. Participants were 1,049 children under five years of age who were hospitalised for severe anaemia. Children were randomised to receive either a three-day course of DP or placebo at weeks two, six, and ten after discharge from hospital (i.e. $3 \times 3$ full DP courses $=9 \mathrm{DP}$ doses). All doses except the first were given at home by the caregiver. All children had received the standard in-hospital treatment for severe anaemia: blood transfusion and parenteral artesunate for those with malaria parasites ( $85 \%$ of the patients). They then received a 3 -day course of artemether-lumefantrine (AL) at the time of discharge. PMC had most impact on malaria outcomes, not readmissions due to other causes. We therefore focus our analysis on incidence of uncomplicated and hospitalised malaria post-discharge, which were measured by passive follow- 
up in study clinics. Malaria cases were hospitalised if they required parenteral treatment, or if they had severe anaemia.

Three further post-discharge studies in Uganda and Malawi were used for model validation, where incidence of hospitalised malaria in children $<5$ years old without chemoprevention was similarly assessed after admission for severe anaemia. ${ }^{10,12,13}$

\section{Post-discharge cohort model}

We developed a compartmental model to describe the incidence of uncomplicated and hospitalised malaria among the trial cohort of children aged 6-59 months during the six months post-discharge period in PMC and placebo trial arms. We related these outcomes to local transmission intensity in the trial sites and rate of antimalarial treatment for symptomatic malaria episodes as well as PMC, to aid extrapolation to other settings. Full details are given in the Supplementary Information. The model has five states: prophylaxis $\left(P_{D P}\right.$ and $\left.P_{A L}\right)$, susceptible $(S)$, treated uncomplicated malaria $\left(T_{U}\right)$, and treated hospitalised malaria $\left(T_{S}\right)$ (Figure 1).

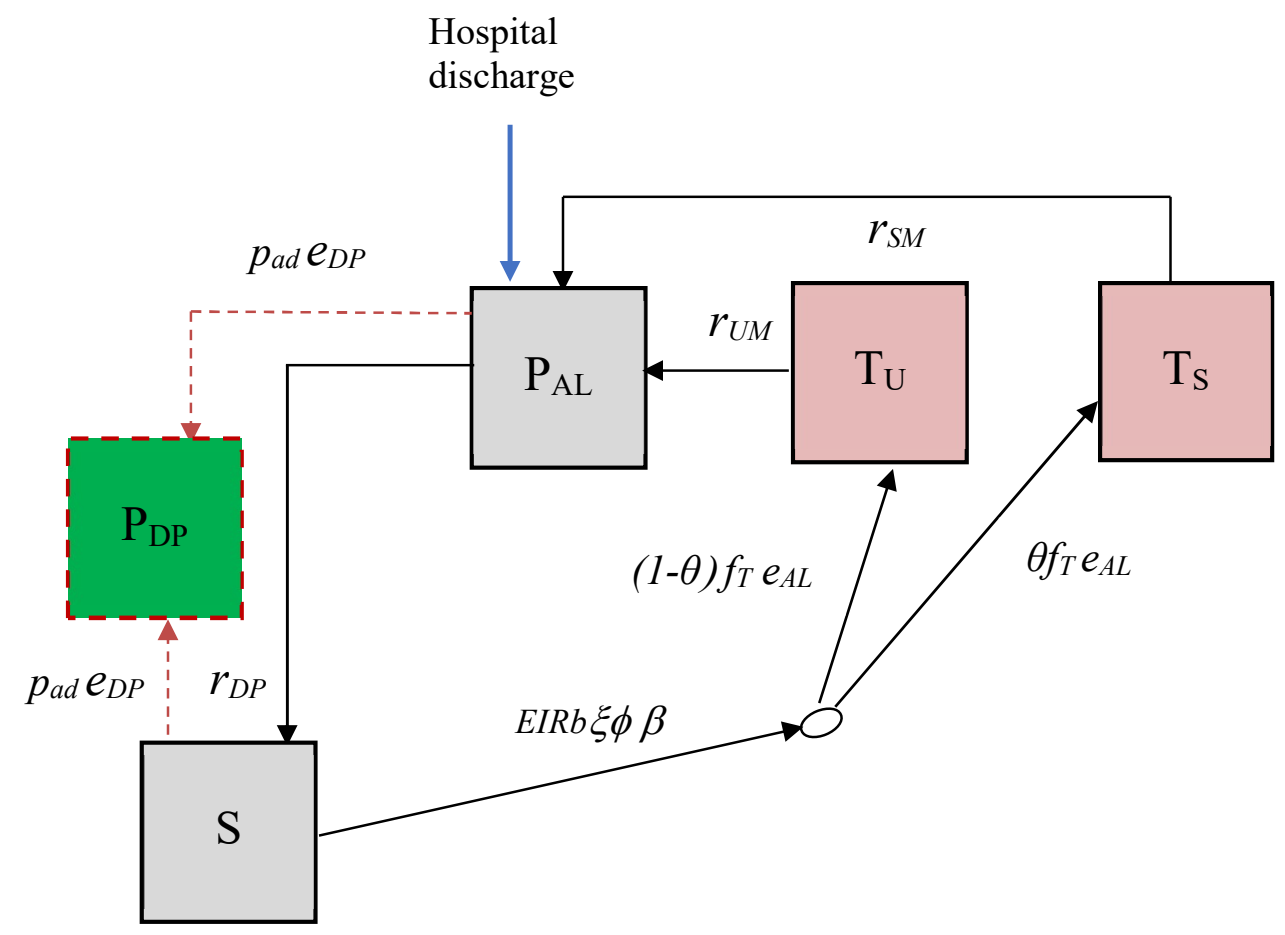

Figure 1: Post-discharge cohort model 
Boxes represent health states, while arrows and labels show health state transition rates and probabilities of an event. Definitions are given in the text and Table S1. See also supplementary information.

Children enter the model without parasitaemia into state $P_{A L}$ on the day they leave hospital, protected from new infections by AL given at discharge. After prophylaxis, children enter the susceptible state and experience symptomatic malaria (uncomplicated or hospitalised) at a rate equal to the product of the local entomological inoculation rate (EIR), the probability that an infectious bite leads to infection $b$, the probability of symptoms $\phi$ and the relative exposure to bites among the post-discharge group of children $\xi$ compared to the average child (Table S1). A proportion $\theta$ of symptomatic malaria cases experience disease sufficiently severe to be readmitted to hospital. We assumed that all uncomplicated and severe malaria episodes during follow-up were detected and treated (at rates $r_{U M}$ and $r_{S M}$ ). This is a reasonable assumption because the trial reimbursed participant costs of treatment-seeking at study clinics, diminishing financial barriers to care. We do not track asymptomatic cases.

Children randomised to the PMC intervention arm receive up to three full courses of DP at the times specified in the trial. DP prophylaxis is modelled as a probability of prevention of reinfection that declines over time since treatment, with a mean of 28 days, as previously estimated (Figure S1). ${ }^{14}$ We allowed for imperfect adherence (Table S1).

\section{Model fitting and validation}

We fitted the model to the trial data described above using Bayesian methods and validated against other post-discharge studies which were not used to fit the model. We used semi-informative priors for the EIR in the areas surrounding each hospital, based on the Malaria Atlas Project (MAP) estimates of transmission intensity within $20 \mathrm{~km}$ (Table S1). ${ }^{15}$ We ran the model for each site and fitted to the daily incidence of uncomplicated and hospitalised malaria simultaneously across sites during 6 months follow up. Since hospitalised malaria numbers were small in some sites, this method borrows information from the uncomplicated malaria cases to inform the underlying EIR. We estimated the maximum incidence of symptomatic malaria (uncomplicated and hospitalised) per infectious bite at the beginning of post-discharge follow up. We estimated how the risk of hospitalised and uncomplicated malaria per infectious bite could decline over time since the original severe anaemia episode, and with increasing EIR. We explored whether the proportion of 
medRxiv preprint doi: https://doi.org/10.1101/2022.01.26.22269679; this version posted January 28, 2022. The copyright holder for this preprint (which was not certified by peer review) is the author/funder, who has granted medRxiv a license to display the preprint in perpetuity.

It is made available under a CC-BY 4.0 International license.

cases that require hospitalisation, $\theta$, was different when the children were or were not under active PMC drug protection. All parameters except for EIR were given weakly informative priors (Table $\mathrm{S} 1)$.

To fit the data, we coded the model in discrete time with a timestep of one day, with transition rates converted to daily probabilities. The model was fitted using Markov chain Monte Carlo methods in the RStan software. ${ }^{16}$ We ran 4 chains, each having 5,000 burn-in and 10,000 sampling iterations. All code is available at https://github.com/lucyokell/pme_model.

\section{Population modelling of PMC demand and impact in different epidemiological settings}

To predict PMC demand and impact in different settings, we developed a model describing severe malarial anaemia (SMA) in the total general population of under-five year olds and embedded our post-discharge cohort model within this framework (full details in the Supplementary Appendix). This model additionally allows for SMA cases who do not seek treatment in hospital, as well as lower treatment coverage for uncomplicated malaria and lower adherence to PMC outside the trial setting. For inputs, we used existing models which relate total population incidence of severe malarial anaemia and hospitalised malaria with EIR and prevalence of infection. ${ }^{21,22}$ We generated estimated annual EIR and incidence of total hospitalised malaria for each sub-national region (first administrative unit) of endemic countries in Africa in 2020 using the Imperial College malaria transmission model. ${ }^{23}$ We estimated incidence of hospitalised SMA in 0-5 year olds in each region based on a recently published analysis of hospital data (Figure S2). ${ }^{22}$

In the full population model (Figure S3), children are stratified into two risk groups: those who experienced SMA within the last 6 months (high-risk) and those who did not (low-risk). When an episode of SMA occurs in low-risk individuals, they move to the high-risk state. The fitted postdischarge cohort model placebo arm was used to describe the incidence of malaria episodes in the high-risk group in the absence of PMC, including SMA episodes. Using estimated total SMA incidence for a given area, the remaining SMA episodes occurring in the low-risk group are calculated. We further stratify SMA cases into those who seek care in hospital versus those who do not. Hospitalised SMA cases are eligible for PMC. After the 6-month period, high-risk individuals return to the low-risk group unless they experience a further episode of SMA. This 
model allows for the iterative process of SMA episodes increasing the risk of future SMA episodes, which affects future PMC impact and demand. For hospitalised SMA episodes and non-SMA malaria episodes, case fatality rates of $7.4 \%$ and $1.0 \%$ were assumed, respectively. ${ }^{17,19,20}$ PMC with DP is incorporated as in the post-discharge cohort model, except that adherence is lower as measured in a recent PMC implementation study ${ }^{18}$ (Table S2).

The probability of malaria patients accessing hospital care when required is challenging to estimate and varies between settings. The relationship between malaria transmission intensity and SMA incidence used in our model is based on hospitalised cases in East Africa, but the probability of hospitalisation in these areas is unknown. ${ }^{22}$ To account for this uncertainty, we varied the probability of hospitalisation between 30,50 , or $70 \%$ for sensitivity analysis ${ }^{24}$ and assumed it could be the same, lower or higher compared to the Paton et al study in different settings. We assumed the case fatality rate of malaria cases unable to access hospital would be double the value observed in hospital. ${ }^{20}$ The model was coded in $\mathrm{R}$ as a deterministic discrete-time model and was run to equilibrium, with and without $\mathrm{PMC}$, to estimate the impact of the intervention. The trials and analyses were approved by the ethics committees at the Kenya Medical Research Institute, Makerere University, the Western Norway Regional Committee for Medical and Health Research Ethics, the Liverpool School of Tropical Medicine, the University of Minnesota, and the Uganda National Council of Science and Technology.

\section{Results}

\section{Post-discharge cohort model}

Our fitted model could capture the total number of observed uncomplicated and hospitalised malaria events in both the post-discharge cohort placebo and PMC trial arms, and the change in daily case incidence over time since discharge (Figure 2). The modelled protective efficacy of PMC, using the previously estimated duration of DP protection of 28 days (Figure S1), was $86 \%$ against hospitalised malaria 3-14 weeks post-discharge and 73\% against uncomplicated malaria during the same period. This was close to the trial estimates of $87 \%$ and $69 \%$, respectively. The differential efficacy against the two outcomes was because the percentage of symptomatic malaria cases that were hospitalised was estimated by the model at 39\% (95\% credible interval $(\mathrm{Crl}) 36$ $42 \%$ ) (Table S1) among children not under active PMC protection, but this dropped to 25\% (95\% 
Crl 17-34\%) among children who had taken recent PMC. The risk of uncomplicated and hospitalised malaria per infectious bite in the placebo group declined over time since discharge (Figure 2 and Figure S4). The incidence of uncomplicated and hospitalised malaria increased with EIR, but the risk of these outcomes per infectious bite decreased (Figures $3 \&$ S5). Three previous post-discharge studies which were not used during model fitting ${ }^{10,12,13}$ showed a similar or slightly lower incidence of post-discharge hospitalised malaria than predicted by our model, given the local EIR (Figure 3B).

The incidence of hospitalised malaria was strikingly high in the post-discharge placebo group compared to the average in the general population of $0-5$-year olds, as estimated in other studies 25 and by our transmission model ${ }^{23}$ (Figure 3B). The incidence of hospitalised malaria in the placebo group was $\sim 39-60$ times higher than in the general population during post-discharge weeks 3-14 in settings with EIR $>10$, and was still 23-36 fold higher in weeks 15-25. This high incidence suggests persistent vulnerability beyond the end of the PMC intervention at week 14 . The incidence of uncomplicated malaria was 1.2-2.5 times higher than expected in the general population of the same age in weeks 3-14. The total incidence of symptomatic malaria episodes (uncomplicated and hospitalised) was more than the expected incidence of infectious bites in 0-5 year olds in four trial sites, suggestive of higher exposure to mosquitoes in this group (Figure S6). 
medRxiv preprint doi: https://doi.org/10.1101/2022.01.26.22269679; this version posted January 28, 2022. The copyright holder for this preprint (which was not certified by peer review) is the author/funder, who has granted medRxiv a license to display the preprint in perpetuity.
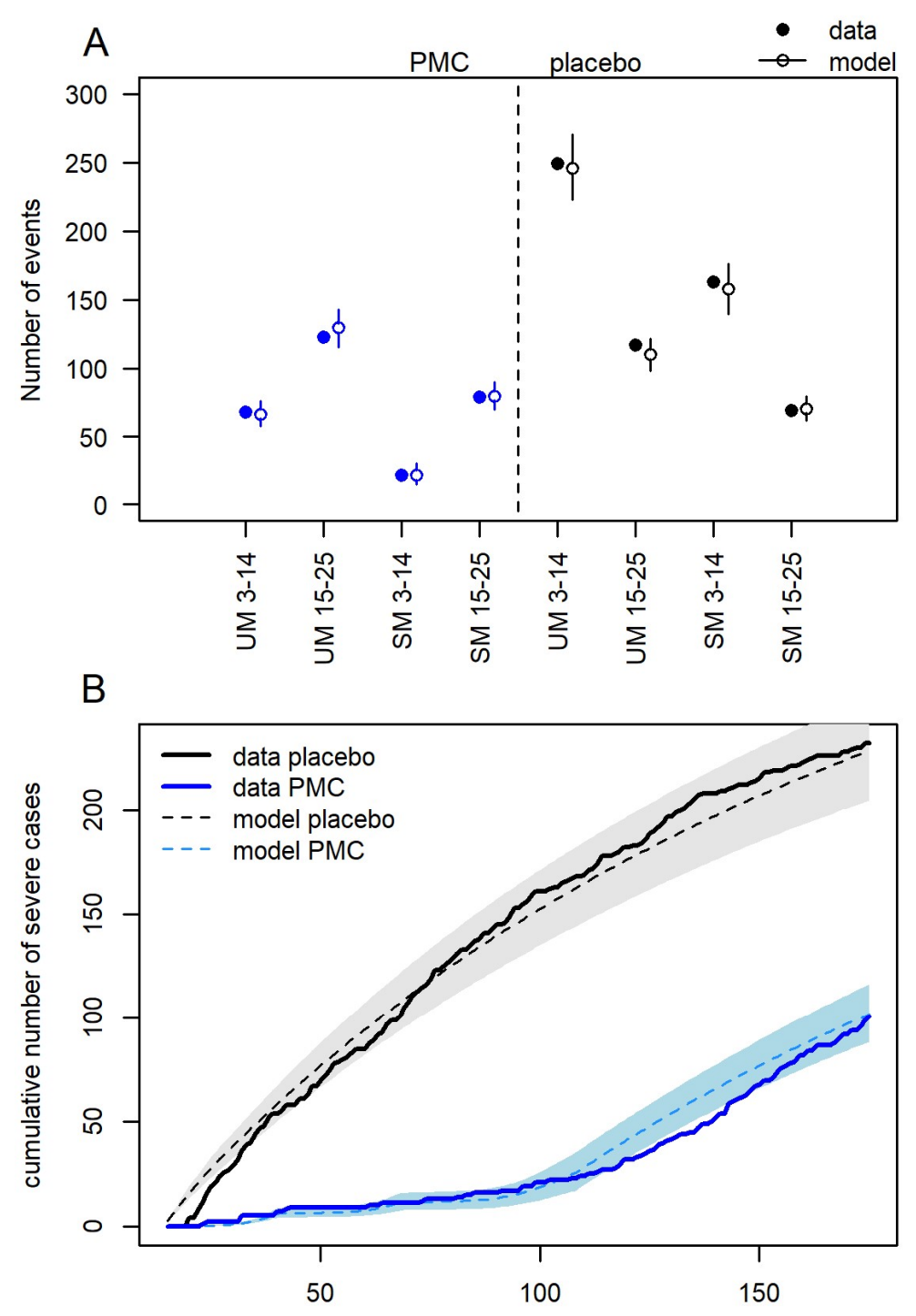

C

days since discharge

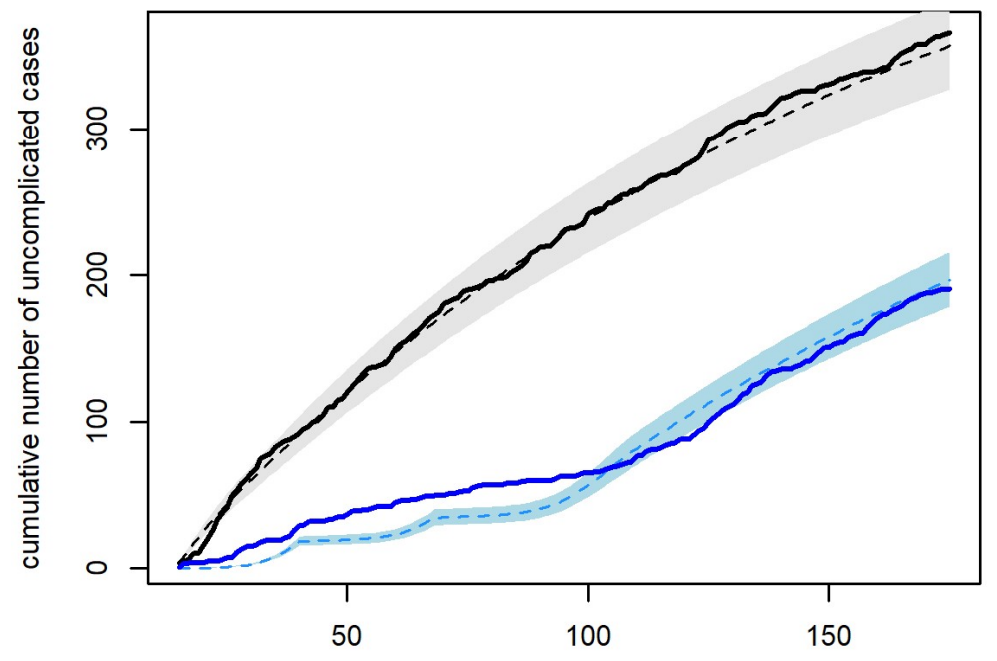

days since discharge 
Figure 2: Model fits to trial data.

Comparison of the number of malaria cases from the trial and model predictions across all sites. (A) Uncomplicated (UM) and hospitalised malaria (SM) in weeks 3-14 and 15-25 post discharge in PMC (left, blue) and placebo (right, black) trial arms. Error bars show 95\% credible intervals ( $\mathrm{Crl})$ of model predictions (B) Cumulative daily number of hospitalised malaria cases by time since discharge after the original SMA episode in placebo (black) and PMC (blue) trial arms; solid line $=$ data, dashed line and shaded area=model fit and 95\% Crl. (C) As B, for uncomplicated malaria cases. See also Figure S7. 

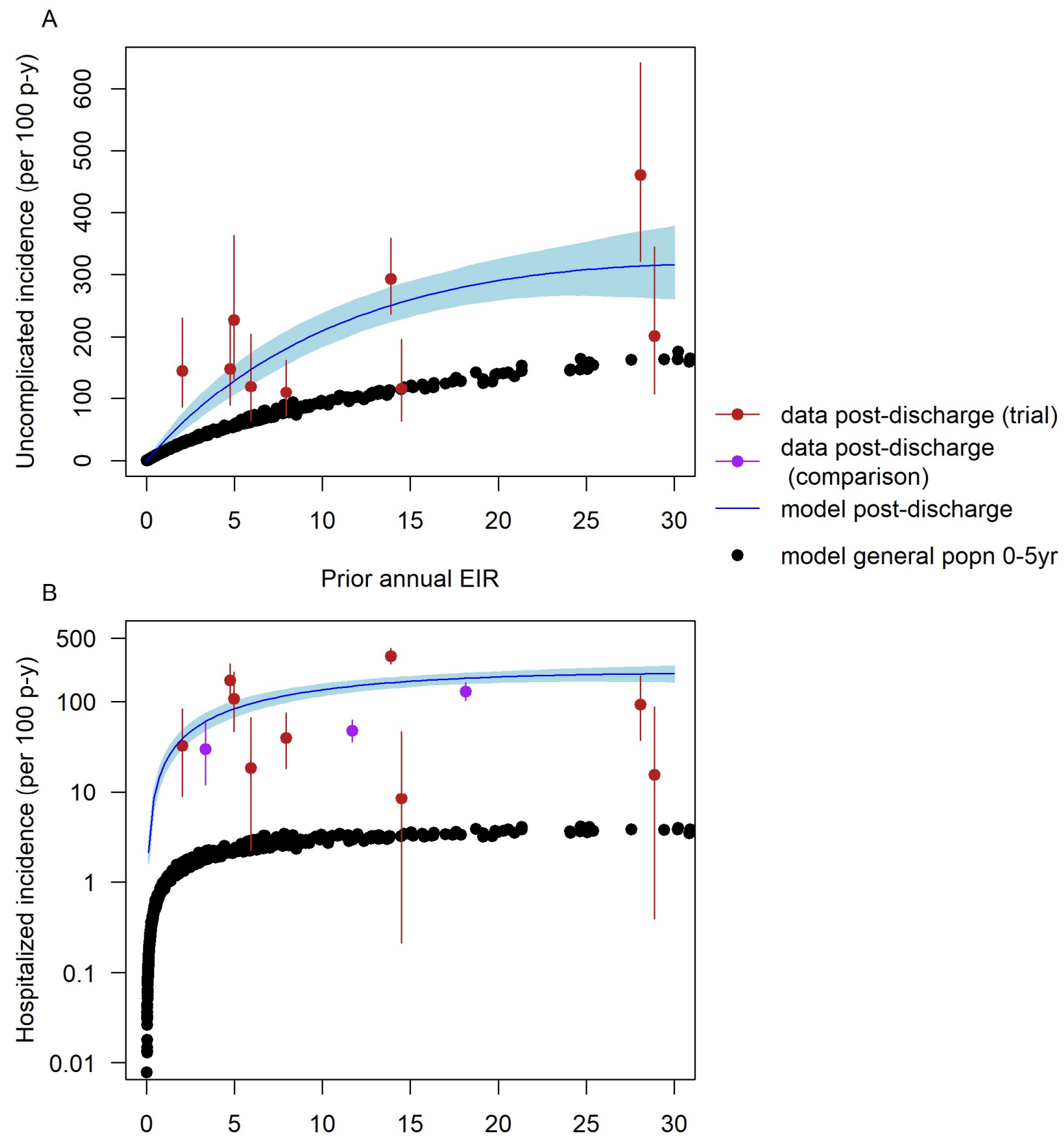

Prior annual EIR

Figure 3 (A) Relationship between prior estimated EIR values in adults and incidence of uncomplicated malaria 3-14 weeks post-discharge in 0-5-year-old severe anaemia patients, per 100 person-years. The data are from the 9 trial locations in Uganda and Kenya (red; data post-discharge (trial) = data from Kwambai et $a l .{ }^{11}$ ). The fitted model (blue; $95 \% \mathrm{Crl}$ ) estimates that the probability of a symptomatic episode per infectious bite declines as the EIR increases (Figure S5). For comparison, the estimated incidence of 
uncomplicated malaria by EIR in the general population of under five-year-olds is shown in black for each subnational region (administrative area 1) in malaria-endemic parts of sub-Saharan Africa. (B) As (A) showing hospitalised malaria episodes in the same trial group (log scale). The model-predicted values are the incidence of hospitalised malaria in the general population of children aged 0-5 years for a given annual EIR. Three additional validation data points are shown from separate post-discharge studies (purple; data post discharge (comparison) in Uganda: Opoka et al. ${ }^{12,13}$ and Malawi: Phiri et al. ${ }^{10}$ which were not used for fitting the model.

\section{Impact of PMC across sub-Saharan Africa and burden of repeat SMA}

The lower adherence to PMC as measured in a PMC implementation study ${ }^{18}$ (Table S2) is estimated to reduce protective efficacy from $86 \%$ to $73 \%$ during weeks $3-14$, resulting in an overall protective efficacy over the 6 months of 39\% (as opposed to 46\% with perfect adherence). The predicted impact of PMC was greatest in countries with higher transmission intensities, where up to 0.45 malaria-associated readmissions could be prevented per child given PMC (Figure 4A). In just over half of the malaria-endemic countries in Africa, we estimate that less than 10 children need to be given PMC to prevent one hospitalised malaria episode (Figure 4B). In the two highest burden countries, Nigeria and the Democratic Republic of the Congo (DRC), ${ }^{5}$ only 3.1 and 2.9 children need to be given PMC to prevent a hospitalised episode, respectively (Table 1).

Predictions of total malaria readmissions and deaths averted were sensitive to assumptions about the proportion of malaria cases who access hospital care, and the case fatality rate outside hospital. The following results assume a base scenario where $50 \%$ of cases requiring hospitalisation actually access it, and a range is obtained from assuming 30-70\% access. If all hospitalised children aged 0-5 years with SMA were given PMC, we estimate that a total of $\sim 36,000$ (range 16,000-82,000) malaria-associated readmissions could be prevented per year across all modelled malaria-endemic countries, and 1,870 deaths prevented per year (range 930-3720). Three-quarters of these prevented episodes are in the ten countries that are the focus of the WHO HBHI programme. ${ }^{5}$ In 23 countries (range 19-24 countries), we estimate that less than 100 children need to be given PMC to prevent one death. In Nigeria and the DRC, 55 (range 28-111) and 51 (range 26-103) children need to be given PMC to prevent one death, respectively. 
A

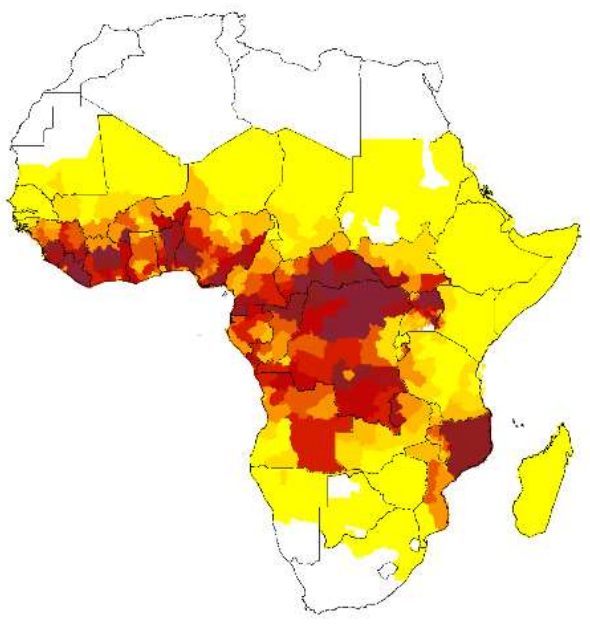

B

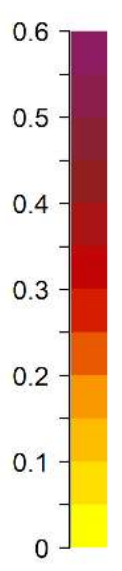

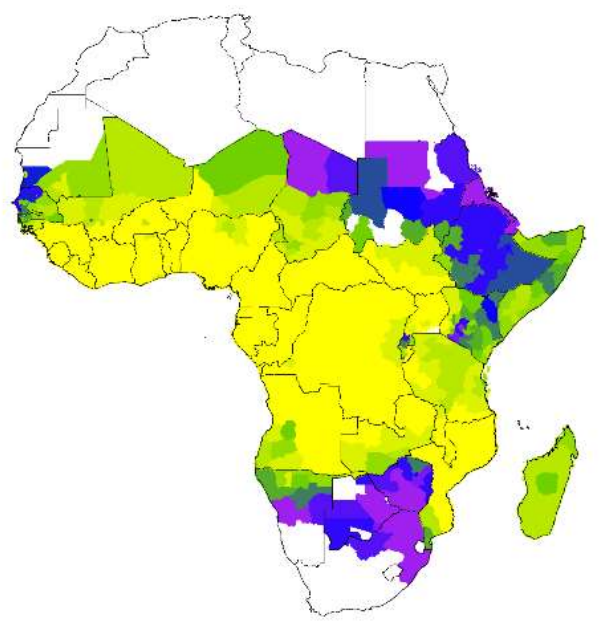

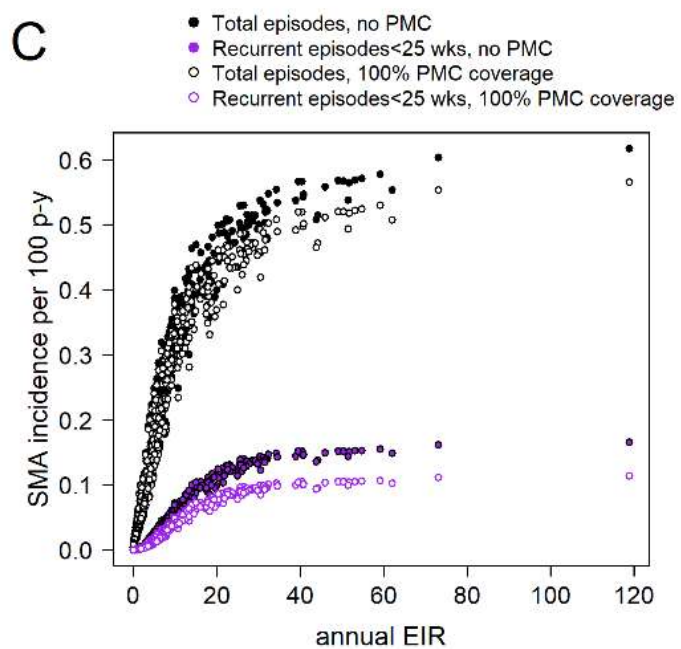

Figure 4. Impact of PMC.

(A) Average number of hospitalised malaria episodes averted per child aged 0-5 years given PMC during the 6 months post-discharge period. (B) Number needed to treat with PMC to avert 1 hospitalised malaria episode. All estimates are shown for subnational (administrative area 1) regions and incorporate imperfect adherence to the three presCrlbed courses of PMC as observed in ref ${ }^{18}$. The assumption in these results is that $50 \%$ of cases requiring hospitalisation access hospital care, but there is negligible change in these outputs when this percentage is varied. (C) Total and recurrent SMA episodes with and without PMC. Model estimates are shown in the absence (solid circles) and presence (open circles) of PMC. Recurrent episodes are those occurring within 6 months of a previous SMA episode. PMC is given at 100\% coverage to hospitalised cases only and impact largely occurs in the first 3 months post-discharge. We allowed for imperfect adherence to PMC. We assumed that 50\% of individuals with SMA reach hospital and varied this for sensitivity analysis (Figure S8). Assuming a low proportion hospitalised results in larger total estimates of SMA burden in the absence of PMC, and smaller total impact of PMC. 
The percentage of all SMA episodes which could be prevented by PMC is highly uncertain, given the unknown probability that cases seek care at hospital in different areas, and that the true burden of disease is therefore unknown. We estimate that in the absence of PMC, the burden of recurrent SMA episodes within 6 months of the original episode is $18-27 \%$ of the total SMA cases in $<5$ year-old children in higher transmission settings with EIR $>10$ (equivalent to slide prevalence in 2-10-year-olds $>25 \%$; Figure 4C), and a lower proportion in lower transmission settings. Weighting by population size, this results in PMC preventing 5-8\% of all SMA in under-fives when EIR $>10$ in the base scenario where $50 \%$ are hospitalised, ranging from 3-11\% under different assumptions about hospital access (Figure S8).

\section{PMC demand forecast}

Using a relationship between EIR and hospitalised SMA incidence based on previous studies in populations with reasonable access to health care, ${ }^{22}$ and assuming a $7.4 \%$ in-hospital case fatality rate, ${ }^{19}$ there would be 123,000 (range 54,000-283,000) SMA survivors under five years old per year across all malaria-endemic countries in Africa (Table 1; Figure S9). The highest number of children eligible for PMC would be in Nigeria with 40,000 per year (range 17,000-94,000). In total, the ten high-burden to high-impact countries identified by $\mathrm{WHO}^{5}$ would require $\mathrm{PMC}$ for 87,000 children per year (range 38,000-201,000). Some countries not on this top-10 list also have high PMC requirements, such as Côte d'Ivoire. 
Table 1: Estimated PMC impact and demand in sub-Saharan African countries. The ten high burden countries targeted by the WHO High Burden to High Impact strategy are shown first. Estimates assume 100\% PMC coverage and the lower adherence level observed in implementation studies

\begin{tabular}{|c|c|c|c|c|c|c|}
\hline Country & $\begin{array}{c}\text { Population } \\
\text { aged under } \\
\text { five years }\end{array}$ & $\begin{array}{c}\text { Incidence of } \\
\text { hospitalised severe } \\
\text { malarial anaemia per } \\
100 \text { person years in 0- } \\
5 \text {-year-olds without } \\
\text { PMC (range*) }\end{array}$ & $\begin{array}{l}\text { Average number } \\
\text { of hospitalised } \\
\text { malaria episodes } \\
\text { prevented per } \\
100 \text { children } \\
\text { given PMC }\end{array}$ & $\begin{array}{c}\text { Number of children } \\
\text { needed to treat with } \\
\text { PMC to prevent } \\
\text { one hospitalised } \\
\text { malaria episode }\end{array}$ & $\begin{array}{l}\text { Number of children } \\
\text { needed to treat with } \\
\text { PMC to prevent one } \\
\text { malaria death } \\
\text { (range })^{\dagger}\end{array}$ & PMC demand (range) \\
\hline \multicolumn{7}{|l|}{$\begin{array}{l}\text { HIGH BURDEN } \\
\text { TO HIGH } \\
\text { IMPACT } \\
\text { COUNTRIES }\end{array}$} \\
\hline Burkina Faso & $3,295,991$ & $0.14(0.1-0.23)$ & 23.5 & 4.3 & $75(67-88)$ & $2,109(919-4,879)$ \\
\hline Cameroon & $4,237,636$ & $0.21(0.15-0.35)$ & 18.8 & 5.3 & $94(83-109)$ & $4,176(1,813-9,660)$ \\
\hline $\begin{array}{l}\text { Democratic } \\
\text { Republic of the } \\
\text { Congo }\end{array}$ & $14,114,883$ & $0.33(0.24-0.54)$ & 34.7 & 2.9 & $51(45-59)$ & $21,369(9,346-49,028)$ \\
\hline Ghana & $4,775,926$ & $0.19(0.13-0.31)$ & 21.5 & 4.6 & $82(73-95)$ & $4,126(1,789-9,500)$ \\
\hline Mali & $3,215,976$ & $0.08(0.06-0.13)$ & 9.6 & 10.4 & $185(163-213)$ & $1,197(520-2,775)$ \\
\hline Mozambique & $4,981,850$ & $0.26(0.19-0.43)$ & 37.6 & 2.7 & $47(42-55)$ & $6,048(2,651-13,857)$ \\
\hline Niger & $3,649,415$ & $0.12(0.09-0.2)$ & 18.9 & 5.3 & $94(82-109)$ & $2,014(869-4,670)$ \\
\hline Nigeria & $33,110,061$ & $0.25(0.18-0.4)$ & 32.1 & 3.1 & $55(49-64)$ & $37,739(16,455-86,551)$ \\
\hline Tanzania & $9,630,285$ & $0.04(0.03-0.07)$ & 8.3 & 12.1 & $215(193-251)$ & $1,834(817-4,406)$ \\
\hline Uganda & $7,112,378$ & $0.2(0.15-0.33)$ & 31.7 & 3.2 & $56(49-65)$ & $6,684(2,920-15,375)$ \\
\hline $\begin{array}{l}\text { OTHER } \\
\text { MALARIA- }\end{array}$ & & & & & & \\
\hline
\end{tabular}




\begin{tabular}{|c|c|c|c|c|c|c|}
\hline $\begin{array}{l}\text { ENDEMIC } \\
\text { COUNTRIES }\end{array}$ & & & & & & \\
\hline Angola & $4,373,828$ & $0.15(0.11-0.24)$ & 21.7 & 4.6 & $82(72-95)$ & $2,992(1,300-6,910)$ \\
\hline Benin & $1,934,664$ & $0.39(0.28-0.64)$ & 37.4 & 2.7 & $48(42-55)$ & $3,502(1,532-8,015)$ \\
\hline Botswana & 288,586 & $0(0-0.01)$ & 0.5 & 215.6 & $3,826(3,937-4,629)$ & $4(2-16)$ \\
\hline Burundi & $2,039,788$ & $0.11(0.08-0.18)$ & 17.6 & 5.7 & $101(90-117)$ & $996(437-2,323)$ \\
\hline $\begin{array}{l}\text { Central African } \\
\text { Republic }\end{array}$ & 880,851 & $0.36(0.26-0.59)$ & 38.7 & 2.6 & $46(40-53)$ & $1,480(648-3,385)$ \\
\hline Chad & $2,552,929$ & $0.06(0.05-0.1)$ & 5.9 & 16.8 & $298(265-347)$ & $726(319-1,720)$ \\
\hline Comoros & 123,614 & $0.03(0.02-0.04)$ & 2.8 & 35.5 & $630(523-707)$ & $15(6-33)$ \\
\hline Cote d'Ivoire & $4,094,415$ & $0.35(0.25-0.57)$ & 34.5 & 2.9 & $51(45-60)$ & $6,560(2,864-15,031)$ \\
\hline Djibouti & 151,672 & $0.01(0.01-0.02)$ & 0.3 & 287.2 & $5,096(4,653-6,168)$ & $5(3-15)$ \\
\hline Equatorial Guinea & 107,080 & $0.45(0.33-0.73)$ & 42.8 & 2.3 & $41(37-48)$ & $221(97-505)$ \\
\hline Eritrea & 950,825 & $0.01(0-0.01)$ & 0.1 & 723.6 & $\begin{array}{c}12,840(14,136- \\
16,176)\end{array}$ & $25(8-77)$ \\
\hline Ethiopia & $18,227,284$ & $0.02(0.01-0.03)$ & 1 & 96.5 & $1,712(1,469-1,889)$ & $1,274(622-3,154)$ \\
\hline Gabon & 288,277 & $0.27(0.2-0.45)$ & 25 & 4 & $71(63-82)$ & $364(158-839)$ \\
\hline Gambia & 332,684 & $0.02(0.02-0.03)$ & 2.5 & 39.9 & $708(661-840)$ & $29(15-73)$ \\
\hline Guinea & $2,233,593$ & $0.23(0.17-0.38)$ & 25.9 & 3.9 & $69(60-79)$ & $2,368(1,028-5,443)$ \\
\hline Guinea-Bissau & 329,360 & $0.04(0.03-0.07)$ & 5.4 & 18.6 & $330(291-384)$ & $62(28-149)$ \\
\hline Kenya & $8,328,699$ & $0.07(0.05-0.11)$ & 22.2 & 4.5 & $80(72-95)$ & $2,568(1,143-6,077)$ \\
\hline Liberia & 788,182 & $0.4(0.29-0.65)$ & 40.8 & 2.5 & $43(38-50)$ & $1,443(632-3,298)$ \\
\hline Madagascar & $4,315,512$ & $0.03(0.02-0.05)$ & 3.7 & 27 & $479(421-552)$ & $632(279-1,512)$ \\
\hline Malawi & $3,123,362$ & $0.12(0.08-0.19)$ & 18.3 & 5.5 & $97(85-112)$ & $1,677(728-3,880)$ \\
\hline Mauritania & 686,348 & $0.06(0.04-0.1)$ & 5.1 & 19.7 & $350(313-409)$ & $191(85-457)$ \\
\hline Namibia & 410,614 & $0.02(0.02-0.04)$ & 3.4 & 29.5 & $523(466-613)$ & $43(19-98)$ \\
\hline Republic of Congo & 830,334 & $0.24(0.17-0.39)$ & 24 & 4.2 & $74(65-85)$ & $919(399-2,117)$ \\
\hline Rwanda & $2,098,732$ & $0.02(0.01-0.03)$ & 1.8 & 56.4 & $1,000(886-1,179)$ & $165(80-441)$ \\
\hline Senegal & $2,623,405$ & $0.02(0.02-0.03)$ & 2 & 49.9 & $886(799-1,063)$ & $218(112-592)$ \\
\hline Sierra Leone & $1,116,213$ & $0.32(0.23-0.52)$ & 39.3 & 2.5 & $45(40-52)$ & $1,661(727-3,798)$ \\
\hline Somalia & $1,915,090$ & $0.04(0.03-0.06)$ & 3.2 & 31.7 & $563(503-657)$ & $318(145-760)$ \\
\hline
\end{tabular}




\begin{tabular}{|l|c|c|c|c|c|c|}
\hline South Africa & $6,646,109$ & $0(0-0.01)$ & 0.2 & 406.6 & $7,214(6,828-8,637)$ & $136(52-493)$ \\
\hline South Sudan & $2,260,515$ & $0.17(0.13-0.29)$ & 20.7 & 4.8 & $86(75-99)$ & $1,813(788-4,196)$ \\
\hline Sudan & $6,139,288$ & $0.01(0.01-0.03)$ & 1.2 & 85.7 & $1,521(1,198-1,815)$ & $400(142-1,006)$ \\
\hline Togo & $1,309,796$ & $0.23(0.16-0.37)$ & 23.5 & 4.2 & $75(66-87)$ & $1,378(598-3,173)$ \\
\hline Zambia & $2,970,088$ & $0.08(0.06-0.13)$ & 19.8 & 5 & $90(79-105)$ & $1,088(476-2,537)$ \\
\hline Zimbabwe & $2,841,665$ & $0.01(0.01-0.02)$ & 0.8 & 119.4 & $2,119(1,841-2,589)$ & $144(70-380)$ \\
\hline TOTAL & & & & & & $\mathbf{1 2 2 , 7 1 3 ( 5 3 , 6 4 1 -}$ \\
& & & & & & $\mathbf{2 8 3 , 2 0 4 )}$ \\
\hline
\end{tabular}

* range obtained from varying the proportion of cases hospitalised from $30-70 \%$.

${ }^{\dagger}$ Children are provided with full PMC ( 3 courses of DP, each containing 3 doses) at discharge, but estimates allow for imperfect adherence in routine settings 


\section{Discussion}

Our model captures the high burden of repeat malaria episodes requiring hospitalisation within the first six months post-discharge among children recovering from an initial episode of SMA. We estimate that this risk is 23-60 times higher than the average for the population of that age. These repeat episodes constitute an estimated one-fifth to one-quarter of all SMA episodes in high transmission settings. PMC with monthly dihydroartemisinin-piperaquine targets this high-risk group and effectively prevents malaria-associated hospital readmissions or death in trials. ${ }^{10,11} \mathrm{We}$ predict that this effect would be notable across most endemic settings in Africa with an effective routine implementation of the intervention. ${ }^{18}$ PMC would have the largest impact in the highest transmission and highest burden settings; e.g. in Nigeria and the DRC, where we estimate only 3 children need to be given PMC to prevent a hospitalised malaria episode. However, we estimate that in most areas fewer than 10 children need to be given PMC to prevent one hospitalised malaria episode. This dynamic is partly driven by the 'self-targeting' nature of PMC - that only high-risk children admitted to hospital will receive the treatment (unlike other forms of chemoprevention, which are administered to all healthy children in a particular age range). Our analysis also suggests PMC is highly cost-effective across a wide range of settings, given that the cost of inpatient malaria care in sub-Saharan Africa is $\$ 15.64-\$ 137.87 .{ }^{26}$ However, cost-effectiveness will depend on the local costs of clinical management and the organisation of health care services.

The risk of hospitalised malaria remained high over a prolonged period, with the incidence still being 23-36 times higher than the average for the age group, 4-6 months post-discharge. Earlier studies suggested that haematological recovery after severe anaemia requires around six weeks. ${ }^{9}$ However, our findings suggest that recently discharged children remain at higher risk than the general population well beyond this period. Since the PMC intervention effect is largely restricted to the first 14 weeks, providing longer protection may have significant health impacts. Future studies should assess the benefit of augmenting PMC with additional prevention strategies against malaria such as longer courses of chemoprevention, malaria vaccines, or monoclonal antibodies that provide at least six months of protection. Caregivers in the PMC trials were encouraged to use insecticide-treated nets, but the provision of a new effective insecticide-treated net might have higher impact. Older children with SMA also have notable post-discharge morbidity, therefore increasing the eligible age range might also increase total impact. 
Our results on the number of cases averted per child given PMC and the number needed to treat to avert adverse outcomes were derived from clinical trial results and are relatively robust to assumptions about hospital access. However, the total demand for PMC and the number of cases and deaths that could be prevented are uncertain, given the lack of data on the probability of accessing hospital care. Access to hospitals is likely to vary greatly between and within countries. ${ }^{20}$ A recent study tracking severely ill children in the community in Uganda, Nigeria, and DRC found that $41-65 \%$ go on to access hospital care. Care seeking for repeat episodes of malaria after hospital discharge during the trial was probably better than in routine clinical practice because participants were financially remunerated for their travel expenses and may have had greater awareness of malaria danger signs. Our analysis finds that PMC has a lower total impact when many children with SMA do not access hospital care. However, we find that the benefit per child given PMC is greater in areas where children are less likely to return to the hospital for subsequent episodes, due to the high burden of repeat severe malaria and its high case fatality rate in the absence of hospital care. This is also important given that poor households can incur catastrophic health expenditure for severe malaria. ${ }^{27}$

Our finding that around $18-27 \%$ of hospitalised SMA episodes would occur within six months of the original episode is plausible considering previous hospital-based studies of children with severe anaemia. In Uganda, $75 \%$ of children with severe anaemia had been hospitalised within the previous six months for SMA. ${ }^{28}$ In the PMC trial in Kenya and Uganda, 35\% of children had been hospitalised previously for severe disease of any cause. ${ }^{11}$ We found that children with SMA are probably exposed to infectious mosquito bites more frequently than the average child, because the incidence of symptomatic malaria post-discharge was higher than the expected incidence of infectious bites in this age group in some settings (Figure S6). The probability of developing infection and symptoms per infectious bite is also higher than in other children. ${ }^{29}$ The group who develop SMA may have particular frailty to malaria for genetic or biological reasons. ${ }^{30}$

Our model was able to replicate the observed total number of hospitalised and uncomplicated malaria episodes in the post-discharge trials. We estimated a relationship between EIR and both outcomes, although there was considerable variation around this in the data. There is uncertainty 
in the EIR values, which are based on predicted prevalence estimates from a geospatial model. ${ }^{15}$ Uncertainty in the incidence of post-discharge episodes also arises from small sample sizes in some hospitals, as well as some misclassification of uncomplicated malaria. One outlier was Kamuli mission hospital in Uganda, which was predicted to have a high EIR yet showed a relatively low incidence of post-discharge hospitalised malaria. This hospital is a private not-for-profit facility, where admitted patients usually incur relatively higher out-of-pocket payments than patients at public hospitals. Trial personnel reported that some malaria cases that would otherwise have been hospitalised were treated as out-patient cases with injectable artesunate to avoid hospital costs and were therefore classified as uncomplicated malaria cases (personal communication, author A. Dhabangi).

In summary, our findings support the widespread implementation of PMC in malaria-endemic countries in Africa, particularly high-burden countries. In countries with low burden, this consideration is likely to be influenced by intervention costs, which may be considered in economic evaluation. However, the total impact of PMC is heavily dependent on the proportion of SMA cases that can access hospital care and the successful delivery of dihydroartemisininpiperaquine. Further, the high risk of malaria morbidity persists for at least six months postdischarge, suggesting the need for longer-acting interventions.

\section{Author contributions}

ATM and LCO: Methodology, Software, Formal analysis, Writing - Original Draft, Visualisation, Writing - Review \& Editing. ACG and MC: Conceptualisation, Writing - Review \& Editing, Funding acquisition. FOTK, KP and BR: Conceptualisation, Writing - Review \& Editing, Project administration, Funding acquisition, Supervision. CK, TKK, AD, TNG, RO, AM, RI, MJK, TCDL and DJW: Data curation, Validation, Investigation, Writing - Review \& Editing.

\section{Declaration of interests}

There are no conflicts of interest to declare.

\section{Acknowledgements}


The study was funded by the Research Council of Norway through the Global Health and Vaccination (GLOBVAC) Programme (project number 234487), which is part of the European and Developing Countries Clinical Trials Partnership (EDCTP2), supported by the European Union through a cooperative agreement with the Liverpool School of Tropical Medicine. LCO is funded by a UK Royal Society Dorothy Hodgkin fellowship and acknowledges funding from the MRC Centre for Global Infectious Disease Analysis (reference MR/R015600/1), jointly funded by the UK Medical Research Council (MRC) and the UK Foreign, Commonwealth \& Development Office (FCDO), under the MRC/FCDO Concordat agreement and is also part of the EDCTP2 programme supported by the European Union; and acknowledges funding by Community Jameel. MC received support from an award (MR/R010161/1) jointly funded by the UK Medical Research Council (MRC) and the UK Department for International Development (DFID) under the MRC/DFID Concordat agreement, which is also part of the EDCTP2 programme supported by the European Union and is supported by a Sir Henry Dale Fellowship jointly funded by the Wellcome Trust and the Royal Society (Grant Number 220658/Z/20/Z). AM acknowledges funding by the UK Medical Research Council (Grant number: G98669, https://mrc.ukri.org/).

\section{References}

1 Casals-Pascual C, Kai O, Cheung JOP, et al. Suppression of erythropoiesis in malarial anemia is associated with hemozoin in vitro and in vivo. Blood 2006; 108: 2569-77.

2 Ippolito MM, Kamavu LK, Kabuya J-B, et al. Risk factors for mortality in children hospitalized with severe malaria in northern Zambia: A retrospective case-control study. Am J Trop Med Hyg 2018; 98: 1699-704.

3 Sawadogo S, Nébié K, Millogo T, Kafando E. Blood transfusion requirements among children with severe malarial anemia: a cross-sectional study in a second level reference hospital in Burkina Faso. Pan Afr Med J 2020; 37: 108.

4 Obonyo CO, Vulule J, Akhwale WS, Grobbee DE. In-hospital morbidity and mortality due to severe malarial anemia in western Kenya. Am J Trop Med Hyg 2007; 77: $23-8$.

$5 \quad$ World Health Organisation. World malaria report 2021. 2021. https://www.who.int/publications/i/item/9789240015791 (accessed Dec 28, 2021). 
6 Madrid L, Casellas A, Sacoor C, et al. Postdischarge mortality prediction in sub-Saharan Africa. Pediatrics 2019; 143: e20180606.

7 Kwambai TK, Mori AT, Nevitt S, Phiri K, Robberstad B, Ter-Kuile F. Post-discharge risks of morbidity and mortality in children admitted with severe anaemia and other syndromes in malaria endemic settings in Africa: A systematic review and meta-analysis. Under review 2022.

8 Phiri KS, Calis JCJ, Faragher B, et al. Long term outcome of severe anaemia in Malawian children. PLoS One 2008; 3: e2903.

9 Price RN, Simpson JA, Nosten F, et al. Factors contributing to anemia after uncomplicated falciparum malaria. Am J Trop Med Hyg 2001; 65: 614-22.

10 Phiri K, Esan M, van Hensbroek MB, Khairallah C, Faragher B, ter Kuile FO. Intermittent preventive therapy for malaria with monthly artemether-lumefantrine for the post-discharge management of severe anaemia in children aged 4-59 months in southern Malawi: a multicentre, randomised, placebo-controlled trial. Lancet Infect Dis 2012; 12: 191-200.

11 Kwambai TK, Dhabangi A, Idro R, et al. Malaria chemoprevention in the postdischarge management of severe anemia. N Engl J Med 2020; 383: 2242-54.

12 Opoka RO, Hamre KES, Brand N, Bangirana P, Idro R, John CC. High postdischarge morbidity in Ugandan children with severe malarial anemia or cerebral malaria. J Pediatric Infect Dis Soc 2016; : iw060.

13 Opoka RO, Waiswa A, Harriet N, John CC, Tumwine JK, Karamagi C. Blackwater fever in Ugandan children with severe anemia is associated with poor postdischarge outcomes: A prospective cohort study. Clin Infect Dis 2020; 70: 2247-54.

14 Okell LC, Cairns M, Griffin JT, et al. Contrasting benefits of different artemisinin combination therapies as first-line malaria treatments using model-based cost-effectiveness analysis. Nat Commun 2014; 5: 5606.

15 Weiss DJ, Lucas TCD, Nguyen M, et al. Mapping the global prevalence, incidence, and mortality of Plasmodium falciparum, 2000-17: a spatial and temporal modelling study. Lancet 2019; 394: 32231.

16 Stan Development Team. RStan: the R interface to Stan. R package version 2.21.2, http://mcstan.org/. 2020.

17 Griffin JT, Hollingsworth TD, Reyburn H, Drakeley CJ, Riley EM, Ghani AC. Gradual acquisition of immunity to severe malaria with increasing exposure. Proc Biol Sci 2015; 282: 20142657.

18 Paton RS, Kamau A, Akech S, et al. Malaria infection and severe disease risks in Africa. Science 2021; 373: 926-31.

19 Griffin JT, Bhatt S, Sinka ME, et al. Potential for reduction of burden and local elimination of malaria by reducing Plasmodium falciparum malaria transmission: a mathematical modelling study. Lancet Infect Dis 2016; 16: 465-72.

20 Mousa A, Al-Taiar A, Anstey NM, et al. The impact of delayed treatment of uncomplicated P. falciparum malaria on progression to severe malaria: A systematic review and a pooled multicentre individual-patient meta-analysis. PLoS Med 2020; 17: e1003359. 
medRxiv preprint doi: https://doi.org/10.1101/2022.01.26.22269679; this version posted January 28, 2022. The copyright holder for this preprint (which was not certified by peer review) is the author/funder, who has granted medRxiv a license to display the preprint in perpetuity.

It is made available under a CC-BY 4.0 International license .

21 Mousa A. The relationship between severe malaria phenotypes and age and estimates of burden across sub-Saharan Africa. Thesis Chapter in prep. .

22 Camponovo F, Bever CA, Galactionova K, Smith T, Penny MA. Incidence and admission rates for severe malaria and their impact on mortality in Africa. Malar J 2017; 16: 1.

23 Nkosi-Gondwe T, Robberstad B, Mukaka M, et al. Adherence to community versus facility-based delivery of monthly malaria chemoprevention with dihydroartemisinin-piperaquine for the postdischarge management of severe anemia in Malawian children: A cluster randomized trial. PLoS One 2021; 16: e0255769.

24 Hetzel MW, Okitawutshu J, Tshefu A, et al. Effectiveness of rectal artesunate as pre-referral treatment for severe malaria in children $<5$ years of age. bioRxiv. 2021; : 2021.09.24.21263966.

25 RTS,S Epidemiology EPI-MAL-002 Study Group. Baseline incidence of meningitis, malaria, mortality and other health outcomes in infants and young sub-Saharan African children prior to the introduction of the RTS,S/AS01E malaria vaccine. Malar J 2021; 20: 197.

26 Conteh L, Shuford K, Agboraw E, Kont M, Kolaczinski J, Patouillard E. Costs and costeffectiveness of malaria control interventions: A systematic literature review. Value Health 2021; 24: $1213-22$.

27 Ilunga-Ilunga F, Levêque A, Laokri S, Dramaix M. Incidence of catastrophic health expenditures for households: an example of medical attention for the treatment of severe childhood malaria in Kinshasa reference hospitals, Democratic Republic of Congo. J Infect Public Health 2015; 8: 13644.

28 Dhabangi A, Idro R, John CC, et al. Risk factors for recurrent severe anemia among previously transfused children in Uganda: an age-matched case-control study. BMC Pediatr 2019; 19: 27.

29 Griffin JT, Hollingsworth TD, Okell LC, et al. Reducing Plasmodium falciparum malaria transmission in Africa: a model-based evaluation of intervention strategies. PLoS Med 2010; 7. DOI:10.1371/journal.pmed.1000324.

30 Valletta JJ, Addy JWG, Reid AJ, et al. Individual-level variations in malaria susceptibility and acquisition of clinical protection. Wellcome Open Res 2021; 6: 22. 